\title{
The generation algorithm of arbitrary polygon animation based on
}

\section{dynamic correction}

\author{
Ya Wei Hou a, Yong Gang Li, Jin Biao Zhou, Jian Wei He and Yu Xin Zhang \\ China Satellite Maritime Tracking and Controlling Department, Jiangyin 214431, Jiangsu, China
}

\begin{abstract}
This paper, based on the key-frame polygon sequence, proposes a method that makes use of dynamic correction to develop continuous animation. Firstly we use quadratic Bezier curve to interpolate the corresponding sides vector of polygon sequence consecutive frame and realize the continuity of animation sequences. And then, according to Bezier curve characteristic, we conduct dynamic regulation to interpolation parameters and implement the changing smoothness. Meanwhile, we take use of Lagrange Multiplier Method to correct the polygon and close it. Finally, we provide the concrete algorithm flow and present numerical experiment results. The experiment results show that the algorithm acquires excellent effect.
\end{abstract}

Key words: Animation, Polygon,Vectors,Correction

\section{Introduction}

As the base of computer animation techniques, two-dimensional animation is a research highlights of current computer science. Two-dimensional animation can be implemented through boundary polygon deformation, so the main mathematical model of two-dimensional animation is polygon deformation techniques. That is to say, in some method to design medium growing change process, the initial object continuously changes to target object on the vision. It is also called 2D-Shape blending. Polygon deformation techniques is widely applied in many fields, such as computer graphics, virtual reality, industry simulation, visualization in scientific computing, bio-medical engineering and so on. And it has already been the important part of computer animation techniques. As an emerging research field, it is with important academic value and research value.

\section{Fundamental assumption and Define}

The deformation process of polygon, without considering the effect of adjacent cycle, need to meet following conditions.

(a) the track of feature points of polynomial(generally the peaks of polynomial) in change period is continuous, smooth and fairing;

(b) the length of polynomial sides is monotonous in change period.

In the process to implement continuous animation, in order to make the polygon continuously transit in different change periods, we need to weaken condition (b) according to requirements, so as to achieve the continuity of deformation track during different periods.

This paper, for the sake of convenience in researching, we give instructions for the following symbols. $\quad A=\left[A_{0}, A_{1}, \mathrm{~L} \mathrm{~L}, A_{n}, \mathrm{~L} \mathrm{~L}\right]$

$T=\left[T_{0}, T_{1}, \mathrm{~L} \mathrm{~L}, T_{n}, \mathrm{~L} \mathrm{~L}\right] \quad$, with

\footnotetext{
${ }^{\text {a }}$ Corresponding author: yaweizq@163.com
} 
$A_{k}=\left\{a_{k, 1}, a_{k, 2}, \mathrm{~L} \mathrm{~L}, a_{k, n}\right\} . A_{k, i}$ means the peak i

of the state polygon $\mathrm{k}$, and record as $\Delta T_{k}:=T_{k}-T_{k-1} \cdot B_{k}(t)$ is continuous deformation sequence of $A_{k-1} \rightarrow A_{k}$. That is to say, the polygon continuous change sequence $(k=1,2,3 . \mathrm{L})$ in interval $\left[T_{k-1}, T_{k}\right]$, in the form of edge vector is $B_{k}(t)=\left\{b_{k, 1}(t), b_{k, 2}(t), \mathrm{L}, b_{k, n}(t)\right\}$.

\section{Second interpolation method of edge-vector}

Define second interpolation vectors of $a_{k-1, s}$ and $a_{k, s}$

$$
b_{k, s}(t)=(1-t)^{2} a_{k-1, s}+2 t(1-t) p_{k, s}+t^{2} a_{k, s} t \in[0,1]
$$

Among them:

$$
p_{k, s}=\left(1+\lambda_{k, s}\right)\left(a_{k-1, s}+\theta_{k, s} a_{k, s}\right) / 2
$$

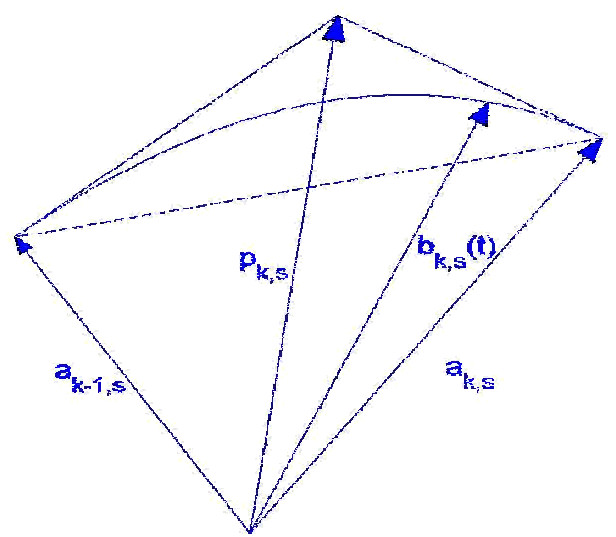

Figure 1 quadratic Bezier interpolation of edge-vector

\section{Dynamic correction of interpolation parameter}

The quadratic Bezier curve defined by the method mentioned above, keeps good characteristics. It only meet the continuation in adjacent periods, while the $\lambda_{k, s} \in(-1, \infty), \theta_{k, s} \in(0, \infty)$

$t$ is the mapping of $\left[T_{k-1}, T_{k}\right]$ in unit interval $[0,1]$.As Fig 1, the physical significance of second interpolation vector defined by (1) is that vector's terminal point track is quadratic Bezier curve of $p_{k, s}-a_{k-1, s} \quad$ and $\quad a_{k, s}-p_{k, s} \quad$ as feature vectors. $p_{k, s}-a_{k-1, s}$ and $a_{k, s}-p_{k, s}$ form feature polygon of interpolation curve.

Vector $b_{k, s}(t)$ keeps good continuity and smooth in deformation period. But in initial deformation period, it is necessary to consider the monotony of vector length. For first-order continuously differentiable curve $b_{k, s}(t)$,it has following theorem:

Theorem 1: the sufficient and necessary condition for $\left|b_{k, s}(t)\right|$ as monotonous on $[0,1]$ is that $\left(b_{k, s}^{\prime}(t), b_{k, s}(t)\right)$ on $[0,1]$ keeps same symbole.

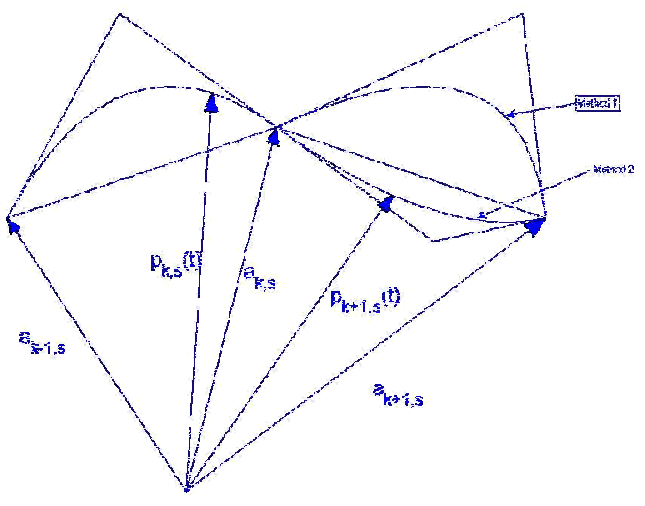

Figure 2 quadratic Bezier interpolation based on dynamic correction

smooth and fairing cannot be realized. Therefor,in order to hold the polygon deformation smooth during animation restructuring procedure, every edge-vector need meet first-order continuity during whole deformation process. That 
is, $\left.\frac{d b_{k-1, s}(t)}{d T}\right|_{T=T_{k-1}}=\left.\frac{d b_{k, s}(t)}{d T}\right|_{T=T_{k-1}}$, according to

Bezier curve characteristic, Bezier curve and its characteristic polynomial's first and last side respectively tangent to the initial and ending points,and the tangent vector is $n$ times of edge-vector.That is:

$$
\frac{2\left(a_{k-1, s}-p_{k-1}\right)}{\Delta T_{k-1}}=\frac{2\left(p_{k}-a_{k-1, s}\right)}{\Delta T_{k}}
$$

It can get from above formula:

$$
\begin{array}{r}
\lambda_{k, s}=\frac{\Delta T_{k-1}}{\Delta T_{k}}\left[2-\left(1+\lambda_{k-1, s}\right) \theta_{k-1, s}\right]+1 \\
\theta_{k, s}=\frac{\Delta T_{k}\left(1+\lambda_{k-1, s}\right)}{2 \Delta T_{k-1}+\Delta T_{k}\left[2-\left(1+\lambda_{k-1, s}\right) \theta_{k-1, s}\right]}
\end{array}
$$

The interpolation curve

$$
\widehat{b_{k-1, s}(t) b_{k, s}(t)} \quad \text { got }
$$

from above formula could meet deformation smooth as $T=T_{k}$. But because of randomness of time sequence, $p_{k, s}$ cannot be guaranteed to fall over the first quadrant of affine coordinate system built by $a_{k, s}, a_{k+1, s}$. So it is necessary to conduct limit to above formula:

$$
\left\{\begin{array}{c}
\lambda_{k, s}=\max \left(\frac{\Delta T_{k-1}}{\Delta T_{k}}\left[2-\left(1+\lambda_{k-1, s}\right) \theta_{k-1, s}\right]+1,-1\right) \\
\theta_{k, s}=\max \left(\frac{\Delta T_{k}\left(1+\lambda_{k-1, s}\right)}{2 \Delta T_{k-1}+\Delta T_{k}\left[2-\left(1+\lambda_{k-1, s}\right) \theta_{k-1, s}\right]}, 0\right)
\end{array}\right.
$$

As shown by Figure 3, the first method is the results by general second interpolation according to Guozhao Wang, and the second method is the results by correction on interpolation parameters according to this algorithm. By comparison, during polygon animation restructuring procedure,the method in this paper could make the transition much smooth and fairing in different periods.

\section{Polygon closure correction}

The polygon formed by above method cannot completely close.That is to say, it is not real sense of polygon. Therefore, we need to amendment the results.
According to the characteristic of polygon,if polygon $B_{k}(t)$ meets the closed condition, it is necessary

$$
\sum_{s=1}^{n} b_{k, s}(t)=0
$$

So, we could make correction on $b_{k, s}$ and add adjustment amount $\eta_{k, s}$ on it, and then as edge-vector of polygon $B_{k}(t)$, that is $b_{k, s}^{*}=b_{k, s}+\eta_{k, s}$

And then we could get:

$$
\sum_{s=1}^{n}\left[b_{k, s}(t)+\eta_{k, s}\right]=0
$$

Define function

$\varphi\left(\eta_{k, 1}, \eta_{k, 2}, \mathrm{~L}, \eta_{k, n}\right)=\sum_{s=1}^{n}\left(b_{k, s}(t)+\eta_{k, s}\right)=0 \quad(4)$

Objective function is $f\left(\eta_{k, 1}, \eta_{k, 2}, \mathrm{~L}, \eta_{k, n}\right)=\sum_{s=1}^{n} \frac{\eta_{k, s}^{2}}{\left|b_{k, s}(t)\right|^{2}}$.It needs to confirm all $\eta_{k, s}$, and make $f$ reach its minuteness under the constrain condition (4).Using Lagrange multiplicator method, take $\lambda$ as multiplicator, and command $\Phi\left(\lambda, \eta_{k, 1}, \eta_{k, 2}, \mathrm{~L}, \eta_{k, n}\right)=f+\lambda \varphi$.

From equation set

$$
\left\{\begin{array}{l}
\frac{\partial \Phi}{\partial \lambda}=0 \\
\frac{\partial \Phi}{\partial \eta_{k, s}}=0
\end{array}\right.
$$

It can be got:

$$
\eta_{k, s}=\frac{b_{k, s}^{2}(t) \sum_{i=1}^{n} b_{k, i}(t)}{\sum_{i=1}^{n} b_{k, i}^{2}(t)}
$$

Therefore, the edge-vector of polygon on time $t$ is 


$$
b_{k, s}^{*}(t)=b_{k, s}(t)+\frac{b_{k, s}^{2}(t) \sum_{i=1}^{n} b_{k, i}(t)}{\sum_{i=1}^{n} b_{k, i}^{2}(t)} 。 \quad(6)
$$

Thus we could calculate polygon $B_{k}(t)$.

\section{Numerical experiment result}

There are many experiments using the method mentioned above. As Figure 4, it is the deformation animation from Chinese character “凹” to a circular. The $1^{\text {st }}, 3^{\text {rd }}$ and $8^{\text {th }}$ frame is polygon key-frames of $\mathrm{T}=0, \mathrm{~T}=0.4, \mathrm{~T}=1.4$. The other frames is polygon reconstructed by the method stated in this paper. We can see from the figure,for the implement of polygon sequence animation with time attributes, polygon gradual changing method based on dynamic correction can achieve the effect of smooth, fairing and natural transition.
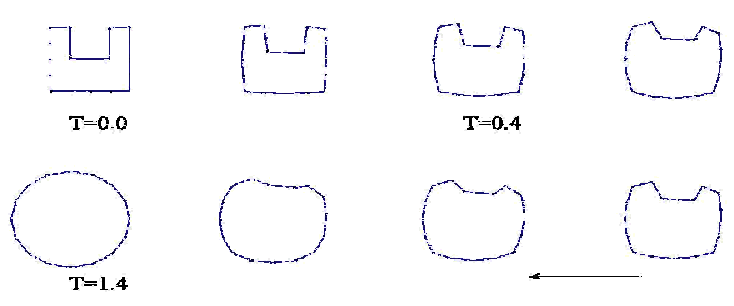

Figure 3 Deforming animation from Chinese character ‘凹' to circular

\section{Conclusion}

This paper proposes one method of polygon animation implement based on dynamic correction. Firstly, vectorize the metamorphosis polygon, and then carry out second interpolation to corresponding edge-vector, meanwhile in the interpolation process, keep first-order continuity of edge-vector and peak-point track, and to the greatest extent maintain the monotony of side.And then, by means of dynamic correction for interpolation parameters, implement continuous transition and fairing in different deformation periods.Finally,correct the deformation edge-vector which uses least square method to close the polygon. Numerical experiment results indicate that the method proposed in this paper implement the smooth and fairing of animation, and the effect is excellent.

\section{References}

[1] Zhao-guo Wang, Bao-gang Bai, Ruo-feng Tong, A vector blending approach for computer animation.J. Chinese Journal of Computers. 12 (1996). 881-886.

[2] LEE T Y, HUANG Pohua. Fast and intuitive metamorphosis of 3D polyhedral models using SMCC mesh merging scheme .J. IEEE Transition Visualization and Computer Graphics, 2003, 9 (1) : 85-98.

[3] The method of computer aided geometric design. Xi'an, Northwestern poly-technical university press, 1986.

[4]Ya-Qing Xu, Bao-gang Bai, UNRBS as a new application of animation vector mixed method. J. Journal of Xinjiang Petroleum Institute 12(2000):69-74 [5] Chang-xu Dou, Yu-mei Wang, Quadratic Interpolation Deformation Algorithm of Polygon Central Point Vector. J. Computer Engineering . 2010.36(16):189-191 